\title{
Tumor Differentiation Factor (TDF) and its Receptor (TDF-R): Is TDF-R an Inducible Complex with Multiple Docking Sites?
}

Urmi Roy, Izabela Sokolowska, Alisa G Woods and Costel C Darie*

Biochemistry and Proteomics Group, Department of Chemistry and Biomolecular Science, Clarkson University, USA

\begin{abstract}
Tumor Differentiation Factor (TDF) is a protein produced by the pituitary and secreted into the blood stream. TDF targets breast and prostate and induces cell differentiation. However, the mechanism of cell differentiation, the TDF receptor and the TDF pathway have not been adequately investigated. Here, we provide some insights about the possible composition of the TDF-R. TDF-R may be a protein complex, composed of GRP78, HSP70 and HSP90 proteins, and all three protein subunits have a docking site for TDF-P1. The question of whether the TDF-R complex is a stable or transient/inducible complex is currently being investigated.
\end{abstract}

Keywords: Tumor differentiation factor; Protein complex; Proteinprotein interactions

\section{Introduction}

Virtually, all expressed proteins in a given cell are arranged into multi-protein complexes [1-9]. Identification of individual components of those complexes is extremely important for their functional characterization $[5,8,10-20]$. One of the most powerful methods in identifying proteins is mass spectrometry, in particular, Liquid Chromatography-Tandem Mass Spectrometry (LC-MS/MS) [5,8,1519]. Combination of LC-MS/MS with a biochemical purification or fractionation strategy makes LC-MS/MS even more powerful, as the protein fractionation allows the LC-MS/MS to increase the number of proteins identified from a particular sample. Affinity PurificationMass Spectrometry (AP-MS), a combination of Affinity Purification (AP) and Liquid Chromatography-Tandem Mass Spectrometry (LCMS/MS), allows for screening of multiple-protein complexes, and for accurate identification of their components $[6,7,9,13,14,21]$. Therefore, a large number of the available Protein-Protein Interactions (PPIs) data, both stable and transient, have been discovered using AP-MS [2,22-26]. Using one protein as bait in AP-MS experiments will usually lead to identification of several potential interactors, and will help to organize them into functional interacting units. Tumor Differentiation Factor (TDF) is a protein produced by the pituitary and secreted into the blood stream. TDF targets breast and prostate and induces cell differentiation. However, the mechanism of cell differentiation, TDF receptor and TDF pathway, have not been thoroughly enough investigated. Here, we provide some insights about the possible composition of the TDF-R, as well as a review of research to date.

\section{Methods}

All biochemical purification and proteomics identification of the TDF-R candidates were performed, as described in [22,23]. All STRING PPIs were performed as in [4,27-31]. All structural biology experiments were performed as in $[3,22,23]$.

\section{Results and Discussion}

Tumor Differentiation Factor (TDF) is a protein produced by the pituitary and secreted into the blood stream [32-35]. The target organs as breast and prostate, and the final effect is cell differentiation [32-34]. Work in our lab also identified TDF in the brain, specifically in neurons, but not in the astrocytes. Additional work in our lab also focuses on identification of the mechanism of TDF-induced cell differentiation. Therefore, some of the questions that we initially asked were 1) what are the potential TDF receptor (TDF-R) candidates? 2) How does TDF-R transduce the differentiation effect across the cell membrane, 3) Is TDF a hormone? To answer to one of these questions, we used TDF-P1, a
20 amino acid peptide from the open reading frame of TDF protein, cross-linked to agarose beads to purify potential TDF-R candidates. In our experiments using DU145 prostate cancer cells and MCF7 breast cancer cells, but not in experiments using HeLa, fibroblasts or other cells, we identified several proteins from the $70 \mathrm{kDa}$ and $90 \mathrm{kDa}$ family of Heat Shock Proteins (HSPs) as TDF-R candidates, with glucoseregulated protein/HSPA5/GRP78, HSP70 and HSP90 being the most likely TDF-R candidates $[7,22,23,34,36]$. Examples of MS/MS spectra that led to the identification of these proteins as TDF-R candidates are shown in Figure 1. The results from our AP-MS experiments could potentially expand the interactome map for those proteins and lead to better understanding of their function in breast and prostate cancer.

To further investigate GRP78, HSP70 and HSP90 proteins as potential TDF-R candidates, and whether these proteins interact with each other and possibly form a protein complex, we have used String database to predict Protein-Protein Interactions (PPIs) and the protein's functional relationships with its partner proteins [28,29,31]. We took dnaK (chaperone HSP70, co-chaperone with DnaJ; Escherichia coli strain K-12 substr. MG1655), $78 \mathrm{kDa}$ glucose-regulated protein (heat shock $70 \mathrm{kDa}$ protein 5 or HSPA5) and HSP90 (heat shock protein $90 \mathrm{kDa}$ alpha) as examples to study their interaction and relation to their functional partners. Network architecture of protein-protein interactions and their functional relatives can be identified and estimated using String. String network (direct and indirect relations) uses several active prediction methods that include "co-expression", "experiments" and "text mining". (Figures $2 \mathrm{~A}$ and $2 \mathrm{~B}, 3 \mathrm{~A}$ and $3 \mathrm{~B}$, and $4 \mathrm{~A}$ and $4 \mathrm{~B}$ ) display possible network of multiple interacting partner proteins (nodes) of dnaK, HSPA5 and HSP90, respectively. A node is the representative of a protein and an edge is the interaction or linkage between two protein partners. Figures $2 \mathrm{C}, 3 \mathrm{C}$ and $4 \mathrm{C}$ are the graphic representation of the observed connectivity between dnaK/GRP78/ HSP90 protein and their ten predicted partners. All these views are in

*Corresponding author: Costel C Darie, Biochemistry and Proteomics Group, Department of Chemistry and Biomolecular Science, Clarkson University, 8 Clarkson Avenue, Potsdam, NY, 13699-5810, USA, Tel: 315-268-7763; Fax: 315268-6610; E-mail: cdarie@clarkson.edu

Received July 23, 2013; Accepted September 03, 2013; Published Septembe 10,2013

Citation: Roy U, Sokolowska I, Woods AG and Darie CC (2013) Tumor Differentiation Factor (TDF) and its Receptor (TDF-R): Is TDF-R an Inducible Complex with Multiple Docking Sites? Mod Chem appl 1: 108. doi:10.4172/23296798.1000108

Copyright: ( 2013 Roy U, et al. This is an open-access article distributed under the terms of the Creative Commons Attribution License, which permits unrestricted use, distribution, and reproduction in any medium, provided the original author and source are credited. 
A

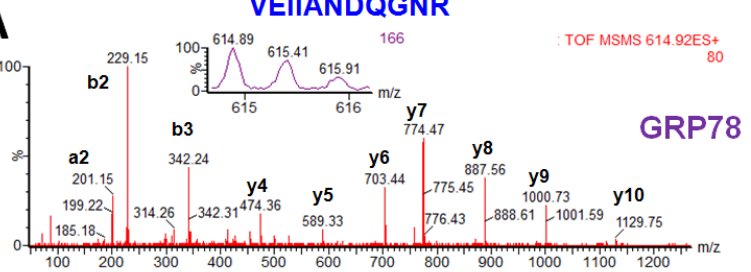

B
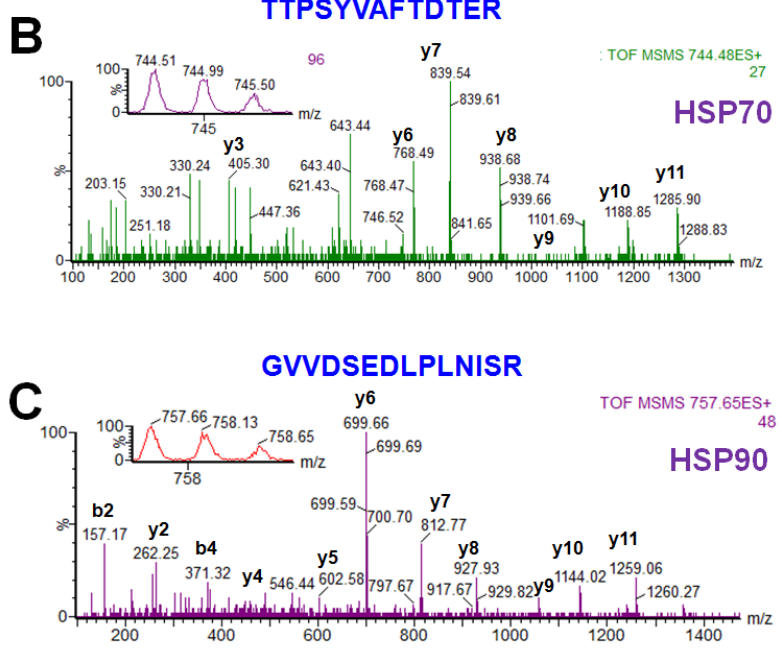

Figure 1: Identification of TDF-R candidates in DU145 cells using AP and LCMS/MS (AP-MS). The potential receptors for TDF protein were purified from cell lysate using AP, resulting samples were separated by SDS-PAGE and the gel bands were excised and digested by trypsin. The peptides mixture was analyzed by LC-MS/MS to identify the purified proteins. A: MS/MS spectrum of peptide VEIIANDQGNR that led to identification of GRP78 as TDF-R candidate. B: MS/ MS spectrum of peptide TTPSYVAFTDTER that led to identification of HSP70 as TDF-R candidate. C: MS/MS spectrum of peptide GVVDSEDLPLNISR that led to identification of HSP90 as TDF-R candidate.

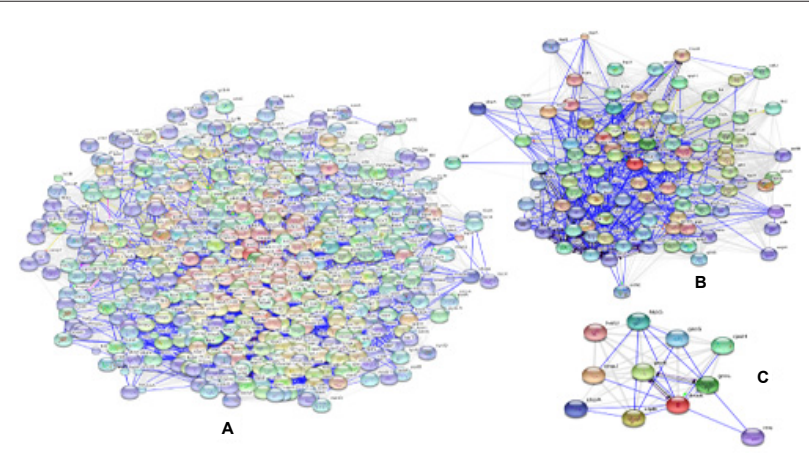

Figure 2: Model interaction network of dnaK chaperone (HSP70) and its possible functional partners. A and B displayed network of approximately five-hundred and one-hundred potential interacting partner proteins (nodes) of dnaK. C. Closer view of interaction. Here the numbers of interacting proteins are ten. These views are in confidence view, where denser lines describe stronger associations. These protein-protein interactions network was generated using STRING program, where a node represents a protein structure and links are projected by edge. The confidence score was set to 0.4 .

action view, where dark lines describe stronger associations. Based on the published results and the String PPI network, it looks indeed as if these three proteins do interact with each other and possibly form a protein complex.

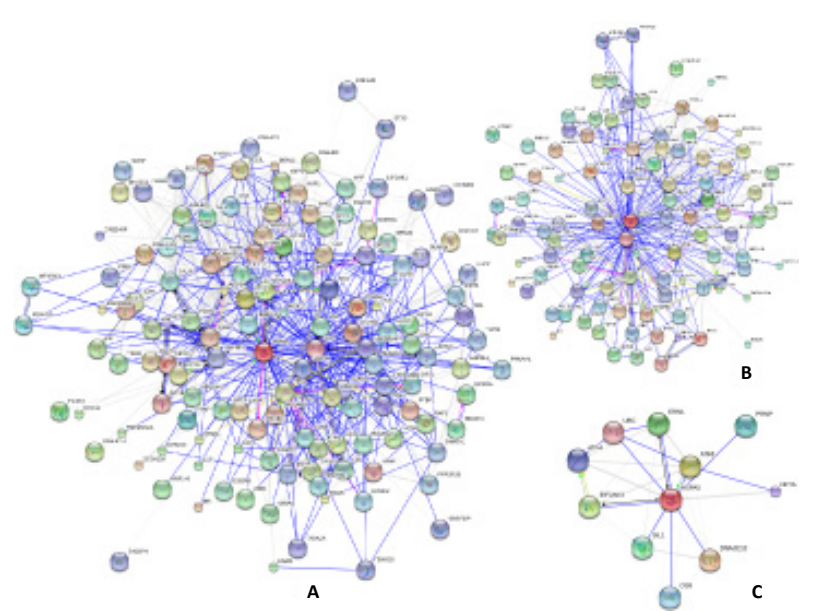

Figure 3: Model interaction network of $78 \mathrm{kDa}$ glucose-regulated protein (GRP78/HSPA5) and its possible functional partners. A and B) Network of multiple potential interacting partner proteins (nodes) of HSPA5. C) Closer view of interaction. Here the numbers of interacting proteins are ten. The darker lines describe stronger associations. These protein-protein interactions network was generated using STRING program, where a node represents a protein structure and links are projected by edge. The confidence score was set to 0.4 .

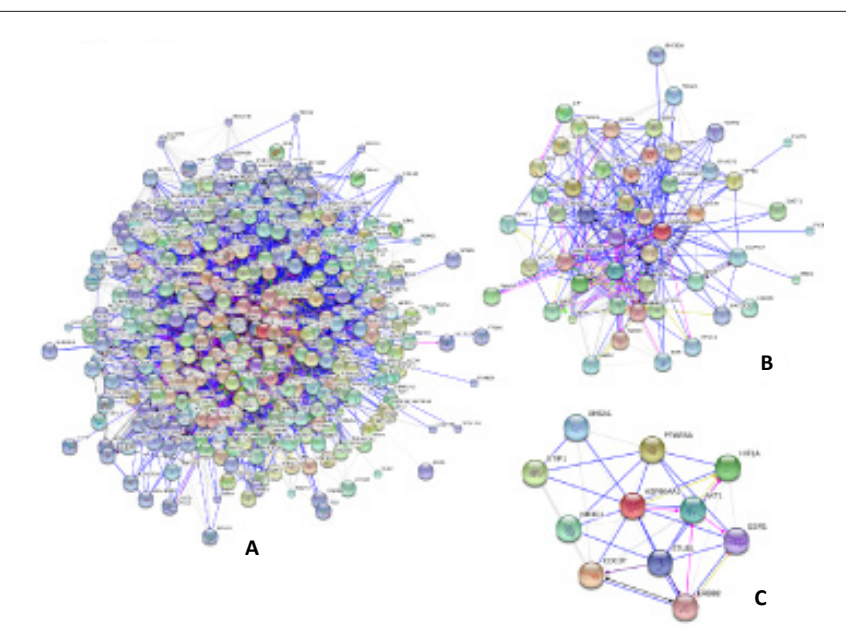

Figure 4: A model protein interaction network of HSP90 (heat shock protein $90 \mathrm{kDa}$ alpha) and its probable functional partners. A and B) Network of approximately five-hundred and one-hundred potential functional partner proteins (nodes) of HSP90. C) Closer view of interaction. Here the numbers of interacting proteins are ten. The thicker lines describe stronger associations. These protein-protein interactions network was generated using STRING program, where a node represents a protein structure and links are projected by edge. The confidence score was set to 0.4 .

Treatment of MCF7 human breast cancer cells and DU145 prostate cancer cells with TDF-P1 leads to differentiation of these cells; this effect is not observed on other non-breast, non-prostate cancer or normal cells $[32,33]$. TDF-P1 is a peptide from the $\mathrm{N}$-terminal part of the TDF that has demonstrated differentiation activity on breast and prostate cancer cells as the full length protein $[32,33]$. Therefore, to interact with TDF-P1 and transduce a differentiation signal, the three TDF-R candidates (GRP78, HSP70 and HSP90) must be present at the cell surface. However, it is still not clear to us whether these proteins form a stable protein complex or is a transient, inducible protein complex. This question is still being investigated in our laboratory. Also, not known is whether the knock down of GRP78, HSP70 and HSP90 will prevent binding of TDF and TDF-P1 to its receptor and will promote cell differentiation. This question is currently investigated in our laboratory. 


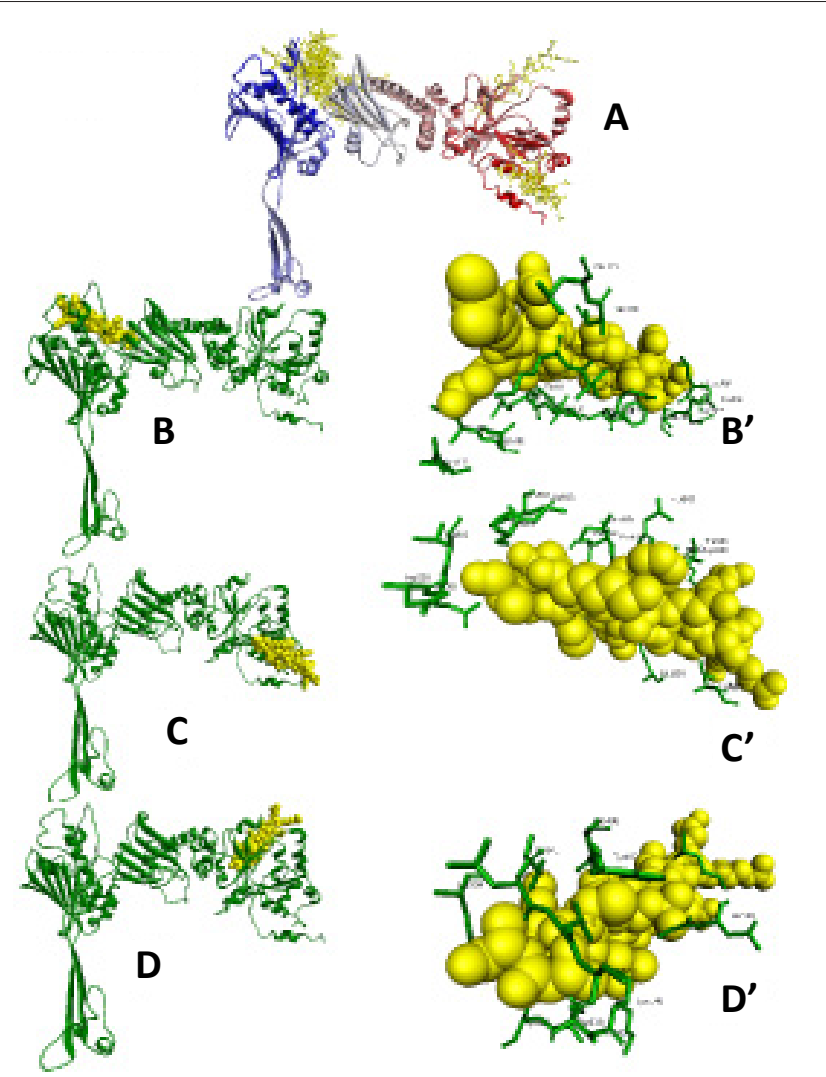

Figure 5: Possible docking sites of TDF-P1 on the model receptor protein as identified by "GRAMM-X". A: Predicted docking sites and poses of docked peptide (yellow). The secondary structure of the model receptor protein is colored from N (blue) to C (red) terminus. B, C and D: Predicted P1 binding sites, with the receptor protein displayed in green ribbon. The $\mathrm{P} 1$ peptide is shown in the yellow space filled mode. B'-D': Neighboring amino acid residues of docked P1 on the model receptor protein

The next question that we asked was whether HSP90, in addition to GRP78 and HSP70, have docking sites for TDF-P1. We already knew that both GRP78 and HSP70 have several docking sites for TDF-P1. Therefore, we investigated HSP90 for possible TDF-P1 docking sites. The crystal structure 2CG9 (chain B, heat shock protein 90-alpha) was used as a template to set up a homology model of HSP 90 [37]. HSP 90 proteins are composed of $\mathrm{N}$ terminal, middle and $\mathrm{C}$ terminal domains. Figure 5A presents the homology model of 2CG9B starting from the $\mathrm{N}$ (colored blue) to $\mathrm{C}$ (colored red) terminals, and the model receptor was established using the SWISS-MODEL server [38,39]. The $\alpha$-carbon Root-Mean-Square Deviation (RMSD) of 2CG9B crystal structure and homology model is $4.13 \AA$ [40]. The docking site of P1 peptide onto the receptor model was identified using the GRAMM-X ProteinProtein Docking Web Server v.1.2.0, as used in our published work $[22,23,35,36,41,42]$. A second run for this identification was carried out using the "Patch dock" and "Fire dock" servers [43-46]. Detailed descriptions of these docking experiments are described in our previous papers $[22,23,35,36]$, and Discovery Studio Visualizer 3.5 was used to plot the tentatively identified binding pockets [47].

We then used structural biology to investigate the possibility that the members of the HSP90 family of proteins are a docking place for TDF-P1. Among the first 10 highest ranked structures developed by "GRAMM-X" web server, P1 was docked onto three regions of the model receptor (Figure 5A). These three potential docking sites and neighboring amino acid residues of P1 peptide are shown in Figures

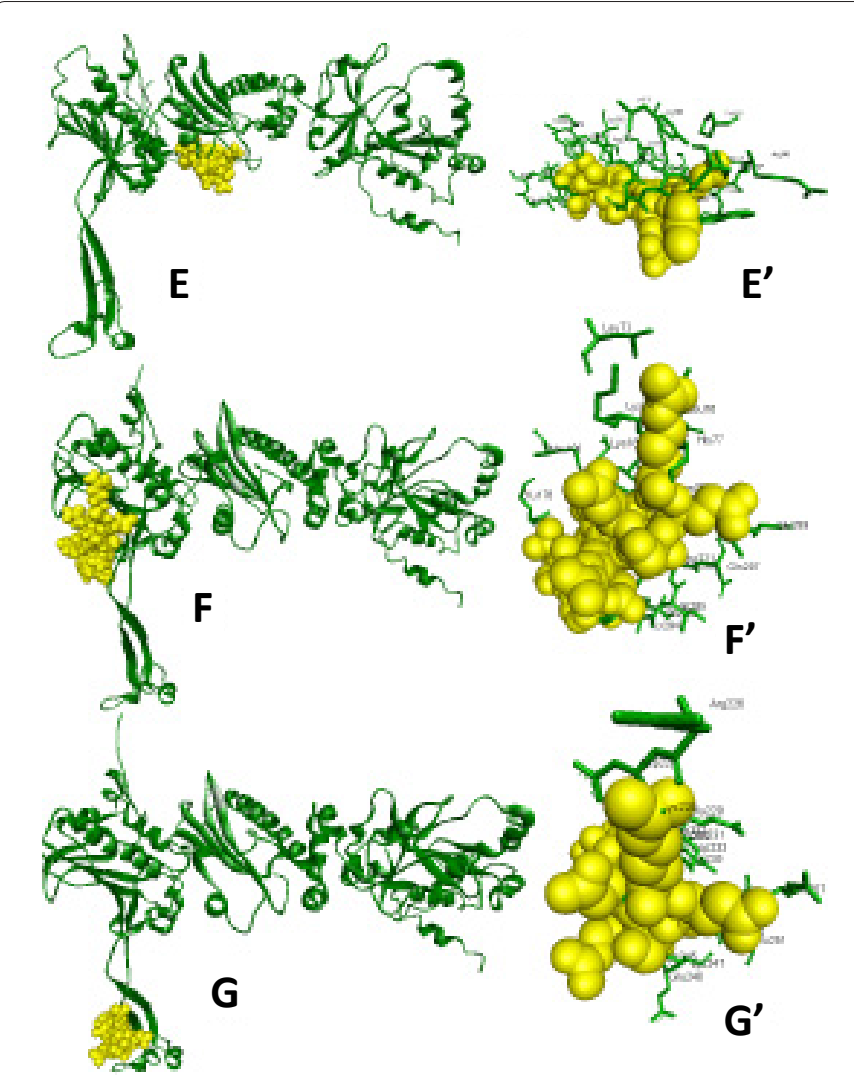

Figure 6: Three additional potential docking sites for TDF-P1, as predicted by using "Patch dock" and "Fire dock".

5B-5D. "Patch dock" and "Fire dock" simulation servers identified three additional potential docking sites for $\mathrm{P} 1$ peptide. These three additional potential docking sites and neighbor residues of docked peptide on the receptor model are shown in Figure 6. Therefore, based on these investigations, our molecular modeling experiments indeed found possible docking sites within HSP90 for TDF-P1.

\section{Conclusions}

Overall, the data allowed us to conclude that the TDF-R may indeed be a protein complex, composed of GRP78, HSP70 and HSP90 proteins, and all three protein subunits have a docking site for TDF-P1. The question of whether the TDF-R complex is a stable or transient/inducible complex is currently being investigated. Current investigations in our laboratory will also allow us to clarify whether there is only one subunit as the main TDF-R, or there is more than one natural docking site for TDF.

\section{Acknowledgement}

Part of this work was started in the Protein Core Facility, Columbia University, New York NY (Dr. Mary Ann Gawinowicz), USA. This work was supported in part by the Keep A Breast Foundation (KEABF-375-35054) and by support from the U.S Army research office through the Defense University Research Instrumentation Program (DURIP grant \#W911NF-11-1-0304). This work was also supported in part by Mary Joyce, Kenneth Sandler, Robert Matloff, and by the SciFund challenge contributors.

\section{References}

1. Ngounou Wetie AG, Sokolowska I, Woods AG, Roy U, Deinhardt K, et al. (2013) Protein-protein interactions: switch from classical methods to proteomics and bioinformatics-based approaches. Cell Mol Life Sci.

2. Ngounou Wetie AG, Sokolowska I, Woods AG, Roy U, Loo JA, et al. (2013) 
Citation: Roy U, Sokolowska I, Woods AG and Darie CC (2013) Tumor Differentiation Factor (TDF) and its Receptor (TDF-R): Is TDF-R an Inducible Complex with Multiple Docking Sites? Mod Chem appl 1: 108. doi:10.4172/2329-6798.1000108

Page 4 of 4

Investigation of stable and transient protein-protein interactions: Past, present, and future. Proteomics 13: 538-557.

3. Ngounou Wetie AG, Sokolowska I, Wormwood K, Beglinger K, Michel TM, et al. (2013) Mass spectrometry for the detection of potential psychiatric biomarkers. J Mol Psychiat 1: 8.

4. Sokolowska I, Dorobantu C, Woods AG, Macovei A, Branza-Nichita N, et al. (2012) Proteomic analysis of plasma membranes isolated from undifferentiated and differentiated HepaRG cells. Proteome Sci 10: 47.

5. Sokolowska I, Gawinowicz MA, Ngounou Wetie AG, Darie CC (2012) Disulfide proteomics for identification of extracellular or secreted proteins. Electrophoresis 33: 2527-2536.

6. Guerrera IC, Kleiner O (2005) Application of mass spectrometry in proteomics. Biosci Rep 25: 71-93

7. Sokolowska I, Woods AG, Wagner J, Dorler J, Wormwood K, et al. (2011) Mass spectrometry for proteomics-based investigation of oxidative stress and heat shock proteins. Oxidative Stress: Diagnostics, Prevention, and Therapy 13 369-411.

8. Woods AG, Ngounou Wetie AG, Sokolowska I, Russell S, Ryan JP, et al (2013) Mass spectrometry as a tool for studying autism spectrum disorder. J Mol Psychiat 1: 6.

9. Woods AG, Sokolowska I, Yakubu R, Butkiewicz M, LaFleur EM, et al. (2011) Blue native page and mass spectrometry as an approach for the investigation of stable and transient protein-protein interactions. Oxidative Stress: Diagnostics, Prevention, and Therapy 12: 341-367.

10. Darie CC (2013) Mass spectrometry and proteomics: Principle, workflow, challenges and perspectives. Mod Chem Appl 1: e105.

11. Darie CC (2013) Investigation of protein-protein interactions by Blue NativePAGE and mass spectrometry. Mod Chem Appl 1: e111.

12. Darie CC (2013) Mass spectrometry and its application in life sciences. Aust $J$ Chem 66: 719-720.

13. Darie CC, Litscher ES, Wassarman PM (2008) Structure, processing, and polymerization of rainbow trout egg vitelline envelope proteins. Applications of Mass Spectrometry in Life Safety 23-36

14. Darie CC, Shetty V, Spellman DS, Zhang G, Xu C, et al. (2008) Blue Native PAGE and mass spectrometry analysis of the ephrin stimulation-dependent protein-protein interactions in NG108-EphB2 cells. Applications of Mass Spectrometry in Life Safety 3-22.

15. Aitken A (2005) Identification of post-translational modifications by mass spectrometry. The Proteomics Protocols Handbook 431-437.

16. Ngounou Wetie AG, Sokolowska I, Woods AG, Wormwood KL, Dao S, et al. (2013) Automated mass spectrometry-based functional assay for the routine analysis of the secretome. J Lab Autom 18: 19-29.

17. Sokolowska I, Ngounou Wetie AG, Roy U, Woods AG, Darie CC (2013) Mass spectrometry investigation of glycosylation on the NXS/T sites in recombinant glycoproteins. Biochim Biophys Acta 1834: 1474-1483.

18. Woods AG, Sokolowska I, Darie CC (2012) Identification of consistent alkylation of cysteine-less peptides in a proteomics experiment. Biochem Biophys Res Commun 419: 305-308

19. Woods AG, Sokolowska I, Taurines R, Gerlach M, Dudley E, et al. (2012) Potential biomarkers in psychiatry: Focus on the cholesterol system. J Cell Mol Med 16: 1184-1195.

20. Thome J, Coogan AN, Woods AG, Darie CC, Häßler F (2011) CLOCK Genes and Circadian Rhythmicity in Alzheimer Disease. J Aging Res 2011: 383091.

21. Darie CC, Deinhardt K, Zhang G, Cardasis HS, Chao MV, et al. (2011) Identifying transient protein-protein interactions in EphB2 signaling by blue native PAGE and mass spectrometry. Proteomics 11: 4514-4528.

22. Sokolowska I, Woods AG, Gawinowicz MA, Roy U, Darie CC (2012) Identification of potential tumor differentiation factor (TDF) receptor from steroid-responsive and steroid-resistant breast cancer cells. J Biol Chem 287 1719-1733.

23. Sokolowska I, Woods AG, Gawinowicz MA, Roy U, Darie CC (2012) Identification of a potential tumor differentiation factor receptor candidate in prostate cancer cells. FEBS J 279: 2579-2594

24. Chautard E, Fatoux-Ardore M, Ballut L, Thierry-Mieg N, Ricard-Blum S (2011) MatrixDB, the extracellular matrix interaction database. Nucleic Acids Res 39: D235-D240.
25. Blagoev B, Kratchmarova I, Ong SE, Nielsen M, Foster LJ, et al. (2003) A proteomics strategy to elucidate functional protein-protein interactions applied to EGF signaling. Nat Biotechnol 21: 315-318.

26. Koh GC, Porras P, Aranda B, Hermjakob H, Orchard SE (2012) Analyzing protein-protein interaction networks. J Proteome Res 11: 2014-2031.

27. Franceschini A, Szklarczyk D, Frankild S, Kuhn M, Simonovic M, et al. (2013) STRING v9.1: protein-protein interaction networks, with increased coverage and integration. Nucleic Acids Res 41: D808-D815

28. Jensen LJ, Kuhn M, Stark M, Chaffron S, Creevey C, et al. (2009) STRING 8-A global view on proteins and their functional interactions in 630 organisms. Nucleic Acids Res 37: D412-D416.

29. http://string.embl.de/

30. von Mering C, Jensen LJ, Kuhn M, Chaffron S, Doerks T, et al. (2007) STRING 7--recent developments in the integration and prediction of protein interactions. Nucleic Acids Res 35: D358-D362.

31. von Mering C, Jensen LJ, Snel B, Hooper SD, Krupp M, et al. (2005) STRING Known and predicted protein-protein associations, integrated and transferred across organisms. Nucleic Acids Res 33: D433-D437.

32. Platica M, Chen HZ, Ciurea D, Gil J, Mandeli J, et al. (1992) Pituitary extract causes aggregation and differentiation of rat mammary tumor MTW9/PI cells. Endocrinology 131: 2573-2580.

33. Platica M, Ivan E, Holland JF, Ionescu A, Chen S, et al. (2004) A pituitary gene encodes a protein that produces differentiation of breast and prostate cancer cells. Proc Natl Acad Sci U S A 101: 1560-1565.

34. Woods AG, Sokolowska I, Deinhardt K, Sandu C, Darie CC (2013) Identification of tumor differentiation factor (TDF) in select CNS neurons. Brain Struct Funct.

35. Roy U, Sokolowska I, Woods AG, Darie CC (2012) Structural investigation of tumor differentiation factor. Biotechnol Appl Biochem 59: 445-450.

36. Sokolowska I, Woods AG, Gawinowicz MA, Roy U, Darie CC (2013) Characterization of tumor differentiation factor (TDF) and its receptor (TDF-R) Cell Mol Life Sci 70: 2835-2848.

37. Ali MM, Roe SM, Vaughan CK, Meyer P, Panaretou B, et al. (2006) Crysta structure of an Hsp90-nucleotide-p23/Sba1 closed chaperone complex. Nature 440: 1013-1017.

38. Arnold K, Bordoli L, Kopp J, Schwede T (2006) The SWISS-MODEL workspace: a web-based environment for protein structure homology modelling. Bioinformatics 22: 195-201

39. Schwede T, Kopp J, Guex N, Peitsch MC (2003) SWISS-MODEL: An automated protein homology-modeling server. Nucleic Acids Res 31: 3381-3385.

40. Maiti R, Van Domselaar GH, Zhang H, Wishart DS (2004) SuperPose: A simple server for sophisticated structural superposition. Nucleic Acids Res 32: W590 594

41. Tovchigrechko A, Vakser IA (2005) Development and testing of an automated approach to protein docking. Proteins 60: 296-301.

42. Tovchigrechko A, Vakser IA (2006) GRAMM-X public web server for proteinprotein docking. Nucleic Acids Res 34: W310-314.

43. Andrusier N, Nussinov R, Wolfson HJ (2007) FireDock: Fast interaction refinement in molecular docking. Proteins 69: 139-159.

44. Duhovny D, Nussinov R, Wolfson HJ (2002) Efficient unbound docking of rigid molecules. Algorithms in Bioinformatics 2452: 185-200.

45. Mashiach E, Schneidman-Duhovny D, Andrusier N, Nussinov R, Wolfson $\mathrm{HJ}$ (2008) FireDock: A web server for fast interaction refinement in molecular docking. Nucleic Acids Res 36: W229-232.

46. Schneidman-Duhovny D, Inbar Y, Nussinov R, Wolfson HJ (2005) PatchDock and SymmDock: Servers for rigid and symmetric docking. Nucleic Acids Res 33: W363-W367

47. Accelrys Software Inc., Discovery Studio Modeling Environment, Release 3.5 San Diego: Accelrys Software Inc., 2012. 